\title{
TURÁN INEQUALITIES FOR SYMMETRIC ORTHOGONAL POLYNOMIALS
}

\section{JOAQUIN BUSTOZ}

Department of Mathematics Arizona State University

Tempe, Arizona 85287

\author{
MOURAD E. H. ISMAIL
}

Department of Mathematics University of South Florida

Tampa, Florida 33620

(Received November 22, 1994)

\begin{abstract}
A method is outlined to express a Turán determinant of solutions of a three term recurrence relation as a weighted sum of squares. This method is shown to imply the positivity of Turán determinants of symmetric Pollaczek polynomials, Lommel polynomials and $q$-Bessel functions.
\end{abstract}

KEY WORDS AND PHRASES. Recurrence relations, Turán determinants, Pollaczek polynomials, Lommel polynomials, $q$-Bessel functions.

1992 AMS SUBJECT CLASSIFICATION CODES. 33C45, 33D45.

\section{INTRODUCTION.}

Let $\left\{P_{n}(x): n=0,1,2, \ldots\right\}$ be , sequence of polynomials orthogonal on an interval $[a, b]$. Then $\left\{P_{n}(x)\right\}$ satisfies a three term recurrence relation

$$
P_{n+1}(x)=\left(A_{n} x+B_{n}\right) P_{n}(x)-C_{n} P_{n-1}(x), n=0,1,2, \ldots .
$$

In (1.1) we take $P_{-1}(x)=0$, and $C_{0}$ is generally unspecified. The polynomials $\left\{P_{n}(x)\right\}$ are said to satisfy Turán's inequality if

$$
\Delta_{n}(x)=P_{n}^{2}(x)-P_{n+1}(x) P_{n-1}(x) \geq 0, a \leq x \leq b .
$$

Paul Turán first noted that (1.2) is satisfied by the Legendre polynomials and Gabor Szegö [15] subsequently gave two elegant proofs of that fact. Since then various authors have proved that (1.2) is satisfied by the classical orthogonal polynomials of Jacobi, Hermite and Laguerre for certain ranges of the parameters involved [9], [14], [15]. A survey of Turán inequalities covering the literature until 1969 is available in [4].

In this note we will prove Turán's inequality for an important class of nonclassical orthogonal polynomials; the symmetric Pollaczek polynomials. The symmetric Pollaczek polynomials satisfy the relation (1.1) with $B_{n}=0$. Thus they satisfy a recursion

$$
E_{-1}(x):=0, E_{0}(x):=1, E_{n+1}(x)=A_{n} x E_{n}(x)-C_{n} E_{n-1}(x), n=0,1,2, \ldots .
$$

We will state a general procedure which makes systematic an approach used by Szàsz in [14] to prove Turán inequalities for ultraspherical polynomials and Bessel functions. In [5] we used Szàsz's method to establish a Turán inequality for the continuous $q$-ultraspherical polynomials. The procedure will be outlined in Section 2. 
Nevai [13] conjectured the positivity of the Turanian for orthonormal Pollaczek polynomials. His conjectures are based on graphing the Turanian for different values of $n$ and his beautiful graphs sparked our interest and led to this work. In Section 2 we apply the Szàsz method to establish the positivity of the Turanian for the Pollaczek polynomials [3], [6], [16]. In Section 3 we establish a Turán inequality for modified Lommel polynomials $\left\{h_{n, v}(x)\right\}$ and $q$-Bessel functions. One can then obtain Szàsz's original result for Bessel functions as a limiting case of the positivity of the Turanian for $q$-Bessel functions. The Hurwitz asymptotic formula for the modified Lommel polynomial, [17, §9.65(1)],

$$
\lim _{n \rightarrow \infty} \frac{h_{n, v+1}(z)}{(2 z)^{n+v} \Gamma(n+v+1)}=J_{v}(1 / z)
$$

will play a central role in our analysis.

\section{THE SZÀSZ METHOD AND APPLICATIONS.}

The idea here is to express $\Delta_{n}$ as a sum of squares by repeatedly using (1.3). Also note that the $P_{n}$ 's of (1.1) or the $E_{n}$ 's of (1.3) are orthogonal if and only if the positivity condition [7, thm I.4.4]

$$
A_{n} A_{n-1} C_{n}>0, n=1,2, \ldots \text {. }
$$

is valid.

THEOREM 1. Let $E_{n}$ be a sequence of polynomials generated by (1.3) and let

$$
g_{n}:=\frac{C_{n}^{2} / A_{n}}{C_{1} C_{2} \cdots C_{n}}, n>1, \zeta_{n}(x)=\frac{C_{n} / A_{n}}{C_{1} \cdots C_{n}}\left[A_{n} E_{n}^{2}(x) / A_{n-1}-E_{n+1}(x) E_{n-1}(x)\right], n>0,
$$

then $\zeta_{n}(x)$ has the finite series representation

$$
\zeta_{n}(x)=\zeta_{m}(x)+\sum_{k=m+1}^{n} g_{k}\left[1-C_{k-1} A_{k} /\left(A_{k-2} C_{k}\right)\right] E_{k-1}^{2}(x), n>m>1
$$

PROOF. Clearly

$$
\begin{aligned}
A_{n} x E_{n}^{2}(x) & =\left[E_{n+1}(x)+C_{n} E_{n-1}(x)\right]\left[A_{n-1} x E_{n-1}(x)-C_{n-1} E_{n-2}(x)\right] \\
& =x A_{n-1}\left[E_{n-1}(x) E_{n+1}(x)+C_{n} E_{n-1}^{2}(x)\right]-C_{n-1} A_{n} x E_{n}(x) E_{n-2}(x) .
\end{aligned}
$$

Divide by $x A_{n-1}$ and rewrite the above when $n \geq 2$ in the form

$$
\begin{aligned}
\frac{A_{n}}{A_{n-1}} E_{n}^{2}(x)-E_{n-1}(x) E_{n+1}(x) & =\frac{C_{n-1} A_{n}}{A_{n-1}}\left[A_{n-1} E_{n-1}^{2}(x) / A_{n-2}-E_{n}(x) E_{n-2}(x)\right] \\
& +C_{n}\left[1-A_{n} C_{n-1} /\left(C_{n} A_{n-2}\right)\right] E_{n-1}^{2}(x) .
\end{aligned}
$$

Next multiply both sides by $C_{n} /\left(A_{n} C_{1} C_{2} \cdots C_{n}\right)$ to reduce the latter identity to

$$
\zeta_{n}(x)=\zeta_{n-1}(x)+g_{n}\left[1-A_{n} C_{n-1} /\left(C_{n} A_{n-2}\right)\right] E_{n-1}^{2}(x), n>1,
$$

which can be iterated to give (2.4) and the proof is complete.

Paul Nevai pointed out that the identity developed in the proof of Theorem 2.2 can be gotten from an identity in a paper of Dombrowski [7]. We have included the derivation of the identity for the sake of completeness and because it is a non-trivial chore to obtain it from Dombrowski's formula.

Theorem 2.2 has two interesting implications. First note that the $g_{k}$ 's have a fixed sign because of the positivity condition (2.1). Indeed $g_{n}$ has the same sign as $A_{0}$, that is

$$
g_{n} A_{0}>0, n>1 \text {. }
$$

There is no loss of generality in always assuming $A_{0}>0$. We will also assume $C_{n}>0, n \geq 1$, since this is the case in all our applications.

COROLLARY 1. Assume that $A_{0}>0$. If $A_{k} C_{k-1} / A_{k-2} C_{k}<1$ for $k>m$ and if $\zeta_{m}(x)>0$ then $\zeta_{n}(x)>0$ for all $n>m$. On the other hand, if $A_{k} C_{k-1} / A_{k-2} C_{k}>1$ for $k>m$ and if $\zeta_{n}(x) \rightarrow 0$ as $n \rightarrow \infty$ then $\zeta_{m}(x)>0$. 
PROOF. The first statement follows immediatcly from (2.4). For the second statement note that if $A_{k} C_{k-1} / A_{k-2} C_{k}>1$ for $k>m$ then from (2.4) we have

$$
\zeta_{n}(x)<\zeta_{m}(x)
$$

Then if $\zeta_{n}(x) \rightarrow 0$ as $n \rightarrow \infty$ we have $\zeta_{m}(x)>0$ and the proof is complete.

The inequality $\zeta_{n}(x)>0$ is not quite of the form (1.2). However it is easy to renormalize the polynomials $E_{n}(x)$ to obtain a Turán inequality like (1.2). To do this, define the monic polynomials $P_{n}(x)$ by

\[ P_{0}(x)=1, P_{n}(x)=E_{n}(x) / A_{0} \cdots A_{n-1}, n \geq 1 . \]
Then the inequality $\frac{A_{n}}{A_{n-1}} E_{n}^{2}(x)-E_{n+1}(x) E_{n-1}(x)>0$ is equivalent to (1.2).

Theorem 1 and Corollary 1 are the essence of the method Szàsz [14] used to establish the positivity of Turanians for ultraspherical polynomials and Bessel functions. An interesting feature of Theorem 1 is that signs of $\zeta_{n}(x)$ and the summand in (2.4) are invariant under renormalization. To see this, replace $E_{n}(x)$ in (2.4) by $c_{n} F_{n}(x)$ so $A_{n}$ and $C_{n}$ need to be replaced by $c_{n} A_{n} / c_{n+1}$ and $c_{n-1} C_{n} / c_{n+1}$, respectively. A simple calculation yields the desired invariance property. Also note that the choice of the coefficient $\dot{A_{n}} / A_{n-1}$ in (2.3) is the only choice that makes $\zeta_{n}(x)$, up to a multiplicative constant, a polynomial of degree $2 n-2$.

As an example consider the continuous $q$-Hermite polynomials $\left\{H_{n}(x \mid q): n \geq 0\right\}[2, \S 6]$, with $|q|<1$. They satisfy (1.3) with $A_{n}=2, C_{n}=1-q^{n}$. They are $q$-analogs of the Hermite polynomials. Thus

$$
g_{k}=\left(1-q^{k}\right) /\left[2(q ; q)_{k-1}\right], \quad 1-A_{k} C_{k-1} / A_{k-2} C_{k}=q^{k-1}(1-q) /\left(1-q^{k}\right),
$$

where we used the notation

$$
(a ; q)_{n}:=(1-a)(1-a q) \cdots\left(1-a q^{n-1}\right), n>0,(a ; q)_{0}=1 .
$$

Therefore

$$
\zeta_{n}(x)=\frac{1-q^{n}}{2(q ; q)_{n}}\left[H_{n}^{2}(x \mid q)-H_{n+1}(x \mid q) H_{n-1}(x \mid q)\right],
$$

so $\zeta_{1}(x)=(1-q) / 2$. Now after some simplification the case $m=1$ of $(2.4)$ reduces to

$$
\frac{\left(1-q^{n}\right)}{(q ; q)_{n}}\left[H_{n}^{2}(x \mid q)-H_{n+1}(x \mid q) H_{n-1}(x \mid q)\right]=\sum_{k=0}^{n-1} \frac{(1-q) q^{k}}{(q ; q)_{k}} H_{k}^{2}(x \mid q) .
$$

The above identity is a Turán inequality for the continuous $q$-Hermite polynomials. The identity (2.13) is a $q$-analog of the Hermite polynomial identity

$$
H_{n}^{2}(x)-H_{n-1}(x) H_{n+1}(x)=2^{n} n ! \sum_{k=0}^{n-1} \frac{1}{k !} H_{k}^{2}(x), n>0 .
$$

We will bricfly show how $H_{n}(x)$ is a limiting case of $H_{n}(x \mid q)$. Since

$$
H_{n+1}(x \mid q)=2 x H_{n}(x \mid q)-\left(1-q^{n}\right) H_{n-1}(x \mid q) \text {. }
$$

then

$$
p_{n}(x):=\left(\frac{2}{1-q}\right)^{n / 2} H_{n}\left(x\left(\frac{1-q}{2}\right)^{1 / 2} \mid q\right)
$$

satisfies

$$
p_{n+1}(x)=2 x p_{n}(x)-\frac{2\left(1-q^{n}\right)}{1-q} p_{n-1}(x)
$$

and 
$p_{0}(x)=1, p_{1}(x)=2 x$. As $q \rightarrow 1$ the above recurrence relation tends to the recurrence relation satisfied by the Hermitc polynomials. This is how one can find the rescaling necessary to go from the continuous $q$-Hermite polyunomials to the Hermite polynomials. We only need to show that $\lim _{q \rightarrow 1} p_{n}(x)$ exists for every $n$. This easily follows by induction using the recurrence relation and initial conditions. A simple calculation now shows how (2.13) tends to the corresponding result for Hermite polynomials as $q \rightarrow 1$.

An important example of Theorem 2.2 is provided by the symmetric Pollaczek polynomials [3, $\S 6],[16]$. They satisfy the recursion (1.3) with

$$
A_{n}=\frac{2(n+a+\lambda)}{n+1}, C_{n}=\frac{n+2 \lambda-1}{n+1} .
$$

The polynomials defined by (1.3) and (2.18) are orthogonal if $\lambda \geq 0$ and $a+\lambda \geq 0$. We find from (2.18) that

$$
1-\frac{A_{n} C_{n-1}}{A_{n-2} C_{n}}=\frac{2[a n+(\lambda-1)(a+\lambda)]}{n(n+a+\lambda-2)(n+2 \lambda-1)} .
$$

The denominator on the right side of (2.19) is positive for $n \geq 3, a+\lambda \geq 0, \lambda \geq 0$. The numerator is positive for large $n$ only where $a>0$, thus we require $a>0$. Taking $m=2$ in (2.4) we have

$$
\zeta_{n}(x)=\zeta_{2}(x)+\sum_{k=3}^{n} g_{k}\left[\frac{1-C_{k-1} A_{k}}{A_{k-2} C_{k}}\right] E_{k-1}^{2}
$$

for $n \geq 3$. For $\zeta_{2}(x)$ we have from (2.3)

$$
\zeta_{2}(x)=\frac{1}{C_{1} A_{2}}\left\{\frac{4(a+\lambda)\left[a \lambda+a+\lambda^{2}-\lambda\right] x^{2}}{3}+\frac{2(a+\lambda+2) \lambda^{2}}{3(a+\lambda+1)}\right\} .
$$

Then $\zeta_{2}(x)>0$ for $-1<x<1$ if

$$
a+a+\lambda^{2}-\lambda>0 \text {. }
$$

When (2.10) holds then $\zeta_{n}(x)>0,-1<x<1, a>0, a+\lambda>0, n=2,3, \cdots$, and then inequality (2.6) holds for the symmetric Pollaczek polynomials. The region defined by (2.10), $a>0, \lambda>0$, in which the Turán inequality (2.6) holds is shown in Figure 1. The region is the area outside the shaded region bounded by the curve $a=\frac{\lambda(1-\lambda)}{1+\lambda}$ and $a>0, \lambda>0$.

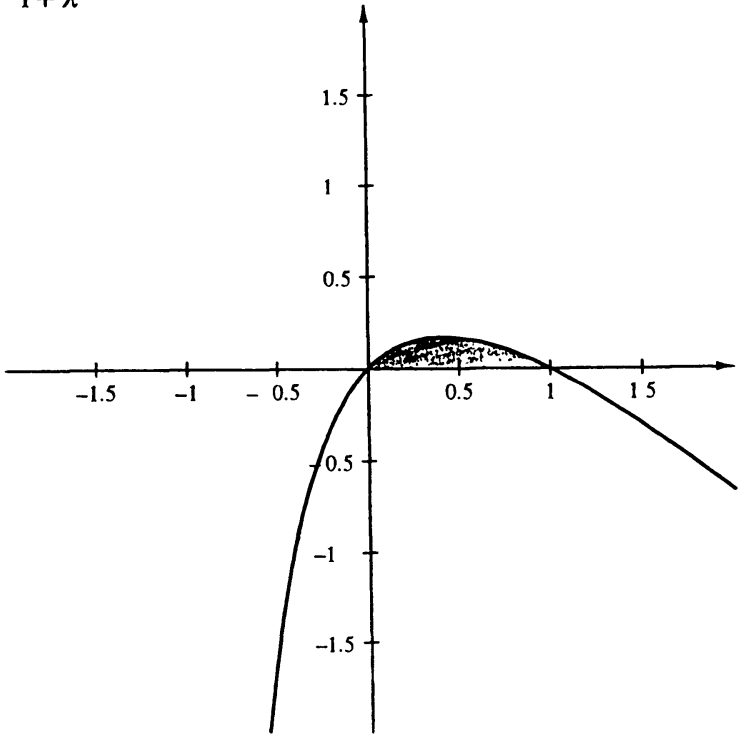




\section{LOMMEL POLYNOMIALS AND $q$-BESSEL FUNCTIONS.}

Dickinson, Pollak and Wanier [8] studied a class of polynomials generated by

$$
p_{-1, v}(x):=0, p_{0, v}(x):=1, p_{n+1, v}(x)=c_{n+v} x p_{n, v}(x)-p_{n-1, v}(x), n \geq 0 .
$$

The positivity condition (2.1) implies that all the $c_{n+v}$ 's have the same sign. Since we may replace $x$ by $-x$ there is no loss of generality in assuming, $c_{n+v}>0$. Later Goldberg [11] corrected a minor error in [8] and proved that if $c_{n+v} \rightarrow \infty$ as $n \rightarrow \infty$ and

$$
\sum_{n=0}^{\infty}\left[1 / c_{n+v} c_{n+v+1}\right]
$$

converges then there exists an even entire function $E_{v}(z)$ such that

$$
\lim _{n \rightarrow \infty} \frac{p_{n, v}(z)}{z^{n} c_{v} c_{v+1} \cdots c_{v+n-1}}=E_{v}(1 / z) \text {. }
$$

Furthermore if

$$
0<x_{1}<x_{2}<\cdots
$$

denote the positive zeros of $E_{v}(z)$ then the polynomials $\left\{p_{n, v}(x)\right\}$ are orthogonal with respect to a discrete measure whose support is $\left\{\frac{1}{x_{n}}: n>0\right\} \cup\{0\} \cup\left\{-\frac{1}{x_{n}}: n>0\right\}$.

The Lommel polynomials $\left\{h_{n, v}(x)\right\}$ correspond to the case $c_{n+v}=2(n+v)[17, \S 9.63(2)]$.

THEOREM 2. Assume that $c_{n+v}$ is monotone increasing. If $c_{n+v+1} / c_{n+v}$ is bounded and the series (3.2) converges then

$$
p_{n, v}^{2}\left(1 / x_{k}\right)-p_{n+1, v}\left(1 / x_{k}\right) p_{n-1, v}\left(1 / x_{k}\right)>\left(1-c_{n+v} / c_{n+v+1}\right) p_{n, v}^{2}\left(1 / x_{k}\right)>0, k=1,2, \ldots .
$$

PROOF. In the present case $A_{k} C_{k-1} / A_{k-2} C_{k}=c_{k+v} / c_{k+v-2}>1$. Also

$$
\zeta_{n}(x)=p_{n, v}^{2}(x) / c_{n+v-1}-p_{n+1, v}(x) p_{n-1, v}(x) / c_{n+v} .
$$

It is known that when the moment problem associated with orthonormal polynomials $\left\{p_{n}(x)\right\}$ has a unique solution then the corresponding measure has a point mass at $x=u$ if and only if $\sum_{n=0}^{\infty} p_{n}^{2}(u)$ converges [1]. In the case under consideration the orthonormal polynomials are $\sqrt{c_{n+v} / c_{v}} p_{n, v}(x)$, hence $\sum_{n=0}^{\infty} c_{n+v} p_{n, v}^{2}\left(1 / x_{k}\right)$ converges for all $k \geq 1$ and $\zeta_{n}\left(1 / x_{k}\right) \rightarrow a$ as $n \rightarrow \infty$. Thus Corollary 1 is applicable and for $n>0$ we have

$$
\zeta_{n}\left(1 / x_{k}\right)=\sum_{m=n+1}^{\infty} \frac{1}{c_{m+\nu}}\left(-1+c_{m+v} / c_{m+\nu-2}\right) p_{m-1, v}^{2}\left(1 / x_{k}\right)>\text { the term } m=n+1,
$$

and (3.5) follows. This completes the proof.

Theorem 2 is the only Turán incquality known for a system of discrete orthogonal polynomials. It is interesting to note that (3.7) holds on the spectrum and may not necessarily hold on the interval of orthogonality $\left(-1 / x_{1}, 1 / x_{1}\right)$. In the case of Lommel polynomials $h_{n, v}(x)$ the $x_{k}$ 's are the positive zeros of $J_{v-1}(z)$. We have calculated the roots of $\zeta_{n}(x)$ when $n=8$ and $v=1 / 2$. When $v=1 / 2$ then $x_{k}=(k-1 / 2) \pi . \zeta_{8}(x)$ is symmetric, of degree 14 , and has four complex roots and 10 real symmetric roots. Denoting the positive real roots by $r_{1}<r_{2}<r_{3}<r_{4}<r_{5}$ we find that

$$
0<\frac{1}{x_{3}}<r_{1}<r_{2}<\frac{1}{x_{2}}<r_{3}<r_{4}<\frac{1}{x_{1}}<r_{5} .
$$

This behavior confirms Theorem 2 . That $1 / x_{1}$ lies between $r_{4}$ and $r_{5}$ becomes numerically evident only when these numbers are calculated to a high level of precision. To 17 places we have 


$$
\begin{aligned}
& r_{4}=.63661942770432881 \\
& \frac{1}{x_{1}}=.636619772367581343 \\
& r_{5}=.6366197723676() 431 .
\end{aligned}
$$

Observe that Hurwitz's formula (1.4) and (3.3) indicate that in the case of Lommel polynomials $\left\{h_{n, v+1}(x)\right\}$ the entire function $E_{v}(z)$ is $\Gamma(v+1)(2 / z)^{v} J_{v}(z)$. Goldberg [11] noted the three term recurrence relation

$$
p_{n, v}(x)=x c_{v} p_{n-1, v+1}(x)-p_{n-2, v+2}(x), n>0 .
$$

If we now let $n \rightarrow \infty$ in (3.9) we obtain from (3.3) the three term recurrence relation

$$
E_{v+1}(x)=E_{v}(x)+\left[x^{2} / c_{v} c_{v+1}\right] E_{v+2}(x) .
$$

If we denote $E_{v+n+1}(z)$ by $F_{n}(z)$ we will find that (3.10) resembles (1.3) with

$$
A_{n}=c_{v+n} c_{v+n+1} / x^{2}, \quad C_{n}=c_{v+n} c_{v+n+1} / x^{3} .
$$

Although now $A_{n}$ and $C_{n}$ depend on $x$ the proof of Theorem 1 remains valid and if $c_{n+v}$ increases with $v$ and the corresponding $\zeta_{m}(x) \rightarrow 0$ as $m \rightarrow \infty$ then

$$
\zeta_{m}(x)>g_{m+1}(x)\left[1-c_{m+v+1} / c_{m+v-1}\right] F_{m}^{2}(x)
$$

which is equivalent to

$$
F_{m}^{2}(x)-F_{m+1}(x) F_{m-1}(x)>0
$$

This establishes the next result.

THEOREM 3. Let $c_{v+n}$ increase with $v$ such that the series (3.2) converges. If

$$
\left[\frac{c_{n+v+1}^{v+n}}{c_{n+v-1}} E_{n+v+1}^{2}(x)-E_{n+v} E_{n+v+2}(x)\right] \prod_{k=1}^{n} \frac{x^{2}}{c_{k+v} c_{k+v+1}} \rightarrow 0
$$

as $n \rightarrow \infty$ then for $x>0$

$$
E_{\nu}^{2}(x)-E_{v+1}(x) E_{\nu-1}(x)>0, v>0 .
$$

Two interesting examples follow from Theorem 3.10. Firstly in the case $c_{v+n}=2(n+v+1)$, $E_{v}(x)=(2 / x)^{v} \Gamma(v+1) J_{v}(x)$ and $E_{v}^{\prime}(x) \rightarrow 1$ as $v \rightarrow \infty$, hence the assumptions in Theorem 3 are fulfilled for $v>0$. Thus (3.11) reduces to

$$
J_{v}^{2}(x)-J_{v+1}(x) J_{v-1}(x)>\frac{1}{v+1} J_{v}^{2}(x), v>0, x>0 .
$$

Szàsz [14] proved (3.16) using recursions. The second example is the case of $q$-Bessel functions of Jackson. Renormalized $q$-Lommel polynomials satisfy (3.1) with

$$
c_{v}=2 q^{1 / 4}\left(q^{-v / 2}-q^{v / 2}\right), E_{v}(x)=(2 / x)^{v} \frac{(q ; q)_{\infty}}{\left(q^{v+1} ; q\right)_{\infty}} J_{v}^{(2)}(x ; q),
$$

where we followed the notation

$$
J_{v}^{(2)}(x ; q):=\frac{\left(q^{v+1} ; q\right)_{\infty}}{(q ; q)_{\infty}} \sum_{n=0}^{\infty} \frac{(-1)^{n}(x / 2)^{v+2 n}}{(q ; q)_{n}\left(q^{v+1} ; q\right)_{n}} q^{n(n+v)},
$$

for Jackson's $q$-Bessel functions [12]. Jackson's original notation was different but the current notation is due to Ismail and since it was introduced in [12] it has become widely used especially after Gasper and Rahman adopted it in their excellent book [10]. The limiting relation (3.14) is satisfied since

$$
\lim _{\nu \rightarrow \infty}(x / 2)^{v} J_{v}^{(2)}(x ; q)=1 /(q ; q)_{\infty},
$$

which follows from (3.18) and the assumption $0<q<1$. Now (3.15) yields the Turán inequality

$$
\left[J_{\nu}^{(2)}(x ; q)\right]^{2}-J_{v+1}^{(2)}(x ; q) J_{v-1}^{(2)}(x ; q)>\frac{q^{\nu}(1-q)}{1-q^{v+1}}\left[J_{v}^{(2)}(x ; q)\right]^{2}, v>0, x>0 \text {. }
$$


It is clear from (3.18) that $(1-q)^{-v} J_{v}^{(2)}(x(1-q): q) \rightarrow J_{v}(x)$ as $q \rightarrow 1^{-}$. The limiting case $q \rightarrow 1^{-}$of (3.20), after replacing $x$ by $(1-q) x$, is the incquality

$$
J_{v}^{2}(x)-J_{v+1}(x) J_{v-1}(x)>\frac{1}{v+1} J_{v}^{2}(x)
$$

mentioned earlier.

ACKNOWLEDGEMENT. This work was partially supported by NSF grants DMS 8814026 and DMS 8912423.

\section{REFERENCES}

1. AL-SALAM, W., ALLAWAY, W., and ASKEY, R. Sieved ultraspherical polynomials, Trans. Amer. Math. Soc. 284 (1984), 39-55.

2. ASKEY, R. and ISMAIL, M. E. H. A generalization of ultraspherical polynomials, in Studies in Pure Mathematics, edited by P. Erdös, Birkhäuser, Basel (1983), 55-78.

3. ASKEY, R. and ISMAIL, M. E. H. Recurrence relations, continued fractions and orthogonal polynomials, Memoirs Amer. Math. Soc., Number 300 (1984).

4. BAJAJ, M. N. The Turán Expressions, M. Sc. Thesis, University of Alberta, Edmonton (1969).

5. BUSTOZ, J. and ISMAIL, M. E. H. Turán inequalities for ultraspherical and continuous $q$ ultraspherical polynomials, SIAM J. Math. Anal. 14 (1983), 807-818.

6. CHIHARA, T. Introduction to Orthogonal Polynomials, Gordon and Breach, New York (1978).

7. DOMBROWSKI, J. Tridiagonal matrix representations of cyclic self-adjoint operators II, Pacific J. of Math. 120 (1985), 47-53.

8. DICKINSON, D. J., POLLACK, H. O., and WANIER, G. W. On a class of polynomials orthogonal over a denumerable set, Pacific J. Math. 6 (1956), 239-417.

9. GASPER, G. On the extension of Turan's inequality to Jacobi polynomials, Duke Math J, 38 (1971), 415-428.

10. GASPER, G. and RAHMAN, M. Basic Hypergeometric Series, Encyclopedia of Mathematics, v. 35, Cambridge (1990):

11. GOLDBERG, J. L. Polynomials orthogonal over a denumerable set, Pacific J. Math 15 (1965), 1171-1186.

12. ISMAIL, M. E. H. The zeros of basic Bessel functions, the functions $J_{v+a x}(x)$, and associated orthogonal polynomials, J. Math. Anal. Appl. 86 (1982), 1-19.

13. NEVAI, P. Private communication.

14. SZÀSZ, O. Inequalities concerning ultraspherical polynomials and Bessel functions, Proc. Amer. Math. Soc. 1 (1950), 256-267.

15. SZEGÖ, G. On an inequality of P. Turán concerning Legendre polynomials, Bull. Amer. Math. Soc. 54 (1948), 401-405.

16. SZEGÖ, G. Orthogonal Polynomials, fourth edition, Colloquium Publications volume 23, Amer. Math. Soc., Providencc (1975).

17. WATSON, G. N. A Treatise on the Theory of Bessel Functions, second edition, Cambridge University Press, Cambridge (1966). 


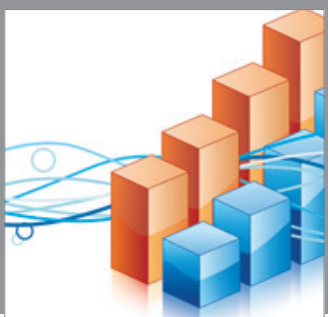

Advances in

Operations Research

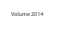

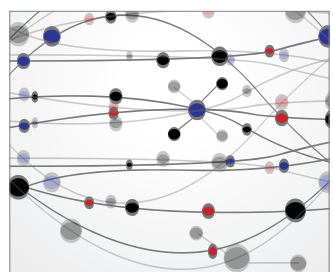

\section{The Scientific} World Journal
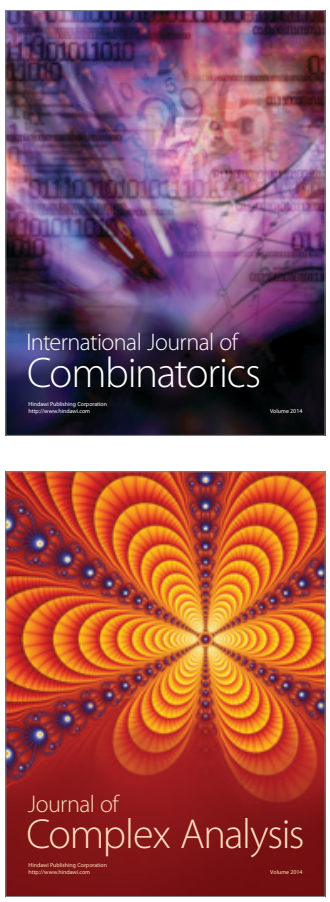

International Journal of

Mathematics and

Mathematical

Sciences
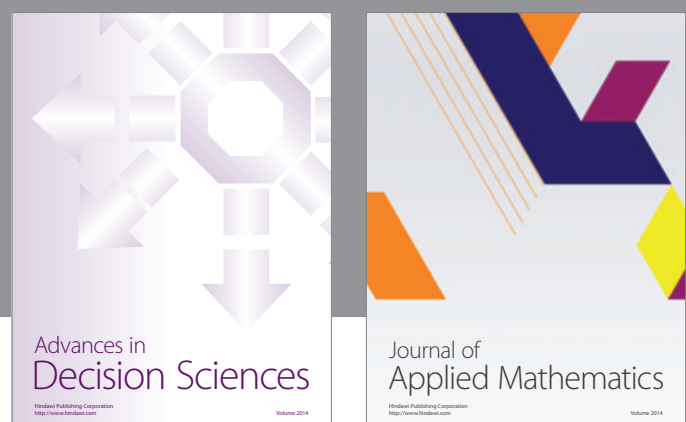

Journal of

Applied Mathematics
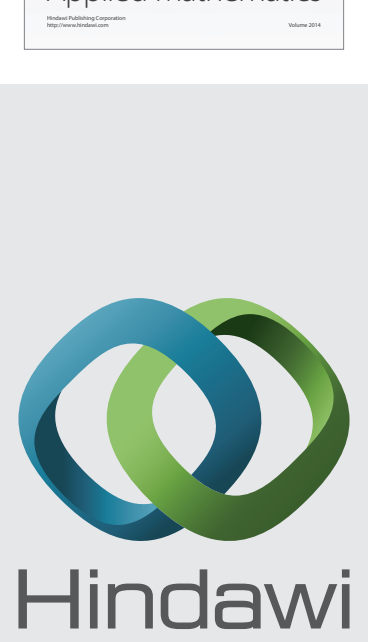

Submit your manuscripts at http://www.hindawi.com
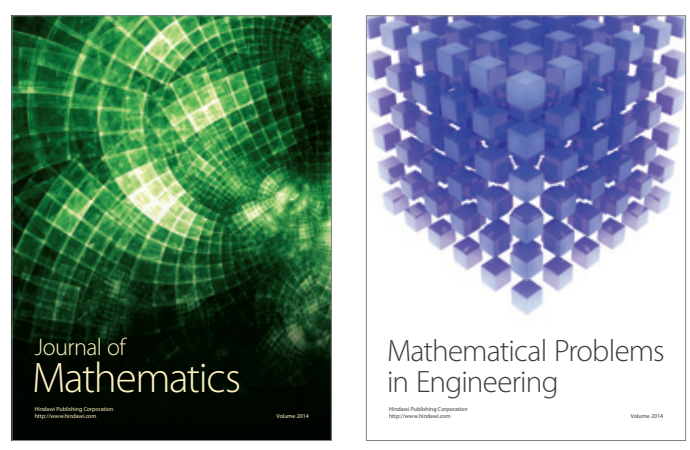

Mathematical Problems in Engineering
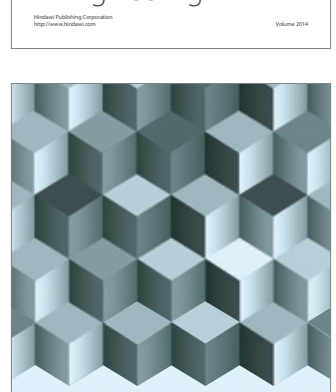

Journal of

Function Spaces
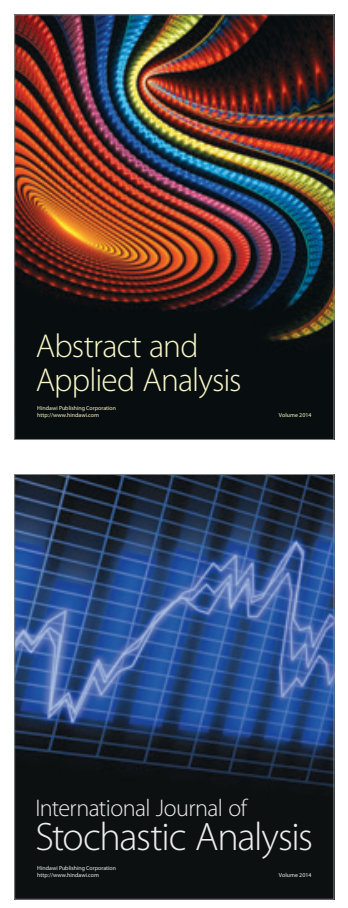

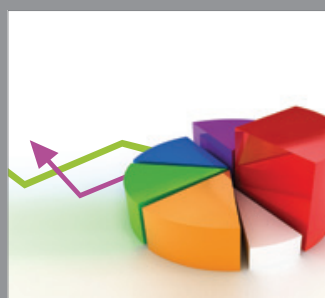

ournal of

Probability and Statistics

Promensencen
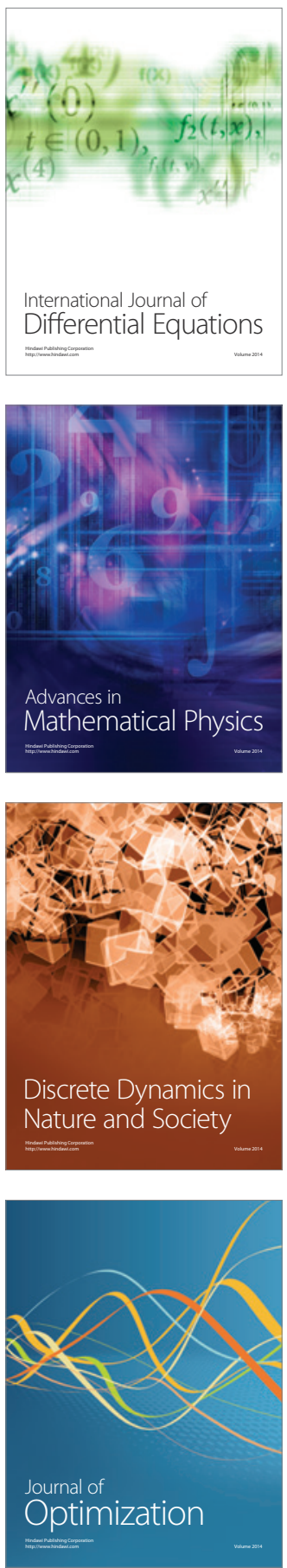